\title{
Comportamiento de variedades de vid resistentes a enfermedades fúngicas en la comarca del Somontano
}

\author{
José Casanova-Gascón $1{ }^{1}$, Carla Ferrer-Martín' ${ }^{1}$, Antonio Bernad-Eustaquio ${ }^{1}$, Andrea Elbaile-Mur ${ }^{1}$, \\ José M. Ayuso-Rodríguez ${ }^{2}$, Sergio Torres-Sánchez ${ }^{2}$, Adrián Jarne-Casasús ${ }^{2}$, Pablo Martín-Ramos ${ }^{1}$ \\ 1 Departamento de Ciencias Agrarias y del Medio Natural, EPS, Universidad de Zaragoza, Carretera de \\ Cuarte, s/n, 22071 Huesca; jcasan@unizar.es. \\ 2 Viñas del Vero S.A., Carretera Nacional 123 (km 3,7), 22300 Barbastro, Huesca.
}

Resumen: La vid es altamente susceptible a numerosas enfermedades de origen fúngico, cuya incidencia y severidad está aumentando como consecuencia del Cambio Climático. Para combatirlas, se ha venido recurriendo al uso de ingentes cantidades de fitosanitarios, los cuales conllevan importantes costes económicos y medioambientales y, en muchos casos, generan resistencias rápidamente. Según datos de Eurostat, la aplicación de fitosanitarios por hectárea y año en viticultura es la más alta de las utilizadas en producción agraria. Las nuevas variedades de vid resistentes a enfermedades fúngicas (denominadas PIWI) constituyen una de las líneas de investigación más activas sobre material vegetal en viticultura, y una solución viable para dar respuesta a las exigencias de la Directiva europea 2009/128/CE. En el presente trabajo se ha realizado un seguimiento de la evolución e impacto de las tres enfermedades principales (mildiu, oídio y botritis) en parcelas de la D.O. Somontano, comparando plantas PIWI (sauvignon kretos, souvignier gris y muscaris) con variedades convencionales como sauvignon blanc en condiciones reales de cultivo durante tres campañas (2016-2018). Se han estudiado los principales caracteres ampelográficos de cada variedad, y se ha realizado un seguimiento semanal en campo del desarrollo de cada enfermedad, relacionándolo con las variables climáticas. Independientemente de las condiciones climáticas (una de las campañas fue lluviosa, y las otras dos correspondieron a años secos, con diferencias en la distribución de las lluvias), las tres variedades PIWI apenas han presentado afección, demostrando una alta resistencia para mildiu y oídio, e incluso para podredumbre gris. En base a los resultados de producción, pese a la juventud de la plantación, se ha observado el excelente potencial de la variedad sauvignon kretos. No obstante, en relación con los análisis de calidad del vino obtenido en microvinificaciones a partir de estos híbridos en la segunda y la tercera campaña, el de la variedad muscaris es el que parece presentar mejores propiedades organolépticas. Desde un punto de vista medio-ambiental, además de contribuir a la sostenibilidad de las bodegas, estas variedades resistentes pueden dar respuesta a la demanda de aquellos consumidores que quieren productos residuo-cero o de los partidarios de productos ecológicos ajustados a la normativa europea.

Palabras clave: Plasmopara viticola, Uncinula necator, Botrytis cinerea, Vitis spp., resistencia, PIWI 


\section{Introducción}

La vid (Vitis vinifera L.) es altamente susceptible a numerosas enfermedades de origen fúngico, principalmente oídio (Uncinula necator), mildiu (Plasmopara vitícola) y podredumbre gris (Botrytis cinerea) [1]. La presión de estas enfermedades está obligando a recurrir al uso de enormes cantidades de fitosanitarios, que comportan importantes costes económicos y medioambientales y en muchos casos generan resistencias rápidamente. Según datos de Eurostat, la aplicación de fitosanitarios por hectárea y año en viticultura es la más alta de toda la producción agraria [2], pues en algunos casos el número de aplicaciones por campaña es superior a 12 [3], llegando a 16 aplicaciones en momentos de elevadas presiones de enfermedad.

La Directiva europea 2009/128/CE establece en su artículo 14 las bases para la utilización de pesticidas de modo sostenible, destacando como aspecto fundamental la reducción de su uso, particularmente el cobre en viticultura [3]. En la legislación española se hace referencia al uso sostenible de los productos fitosanitarios en el RD 1311/2012. Una de las estrategias más prometedoras pasa por un tratamiento de prevención basado en el desarrollo de variedades resistentes a hongos [3].

La selección asistida con marcadores, combinada con retrocruzamientos con múltiples variedades de $V$. vinífera, ha permitido el desarrollo de variedades de uva resistentes a hongos con dos genes de resistencia y un porcentaje significativo (más del 85\%) de $V$. vinifera en su pedigrí: las conocidas como "PIWI" (del alemán Pilzwiderstandsfähig, "resistente a enfermedades fúngicas"), y que a día de hoy son aceptadas como $V$. vinifera en los catálogos europeos [4]. En el Reglamento (UE) 1308/2013 se recoge la utilización de estas variedades PIWI para la elaboración de vinos, siempre y cuando estén previamente inscritas en el Registro de variedades del país pertinente.

La resistencia de estas variedades varía con la genética del cultivar y la localización [5]. Por tanto, la mayoría de los cultivares de variedades resistentes muestra cierta susceptibilidad a diferentes patógenos, incluidos oídio, mildiu, botritis, pudrición negra y antracnosis. Estas enfermedades son controladas generalmente con fungicidas a base de azufre [3,6] o con formulaciones basadas en cobre, pero con un número de aplicaciones mucho menor que en variedades tradicionales [7]. En un estudio realizado en 6 países europeos, que incluyó 183 variedades PIWI, se obtuvo como resultado una reducción del número de tratamientos fungicidas entre un $73 \%$ y un $82 \%$ para grados de presión de la enfermedad bajo y medio, respectivamente [3]. Un resultado similar fue también obtenido en un estudio en el que participaban 65 viñedos alemanes bajo manejo ecológico, cuyos productores informaron que pulverizaban las variedades resistentes a hongos unas 3,8 veces de promedio por campaña [8].

Se espera que las PIWI supongan un ahorro de costes muy importante en el control anual de las enfermedades: en Francia, se ha estimado que la producción con variedades resistentes podría llegar a disminuir a la mitad los costes de producción [9]; y en California se ha previsto que la utilización de variedades resistentes a oídio podría suponer un ahorro de hasta 48 millones de dólares por año en la producción de uva de mesa, pasas y vino [10].

Respecto al rendimiento, las variedades resistentes generalmente son más vigorosas y muestran mayor productividad [3,11-13].

Pese a las ventajas preconizadas, las variedades resistentes a hongos se encuentran prácticamente ausentes del mercado vitivinícola de la mayoría de los países productores por la injustificada desconfianza tanto sobre su utilización [8] como sobre la calidad enológica del vino. Esta surgió a cuenta del hecho de que las PIWI portan genes no- $V$. vinifera (incluso a niveles bajos), que llevó a la presunción de que podían dan lugar a vinos de menor calidad [10]. Actualmente, existen estudios que demuestran que la calidad de los vinos procedentes de variedades resistentes a hongos es generalmente equivalente a los producidos a partir de $V$. vinifera $[3,7]$.

En España, los únicos campos de ensayo experimentales con PIWI, además del de Viñas del Vero -en el que se ha realizado el presente trabajo-, son uno existente en Olite y otro en el Valle 


\section{CONGRESO IBÉRICO DE AGROINGENIERÍA \\ X CONGRESSO IBÉRICO DE AGROENGENHARIA \\ 3 - 6 septiembre 2019, Huesca - España}

de Baztán en Navarra [14]. Todos ellos fueron plantados en 2015, y no se han hecho públicos datos de producción y/o uso de fitosanitarios.

En la presente comunicación se recogen los resultados para tres variedades PIWI (sauvignon kretos, souvignier gris y muscaris) durante las campañas 2016-2018, en un estudio pionero en nuestro país.

\section{Material y métodos}

\subsection{Localización}

El viñedo experimental se localiza en una parcela propiedad de Viñas del Vero S.A., en el término municipal de Barbastro, partida 'Las Almunietas'. Las coordenadas UTM de la parcela son: $X=261706, Y=4652417$, huso 31.

Respecto a la edafología, en Badía, et al. [15], se recoge un estudio detallado del suelo de la zona de 'Las Almunietas', concretamente el que hace referencia a 'Torre Fierro'. Esta zona está formada por gipsisoles, suelos desarrollados sobre margas yesíferas que presentan una acumulación secundaria de yeso en formas pulverulenta y/o lenticular a cierta profundidad. Son suelos de texturas francas, pedregosidad escasa, capacidad de retención de agua disponible moderada y capacidad de intercambio catiónico baja.

\subsection{Material vegetal}

El material vegetal que conforma el estudio son tres variedades PIWI blancas y una variedad control (sauvignon blanc). La primera variedad PIWI, sauvignon kretos (C.v. 76-026), fue obtenida por el Instituto de Genómica Aplicada de Udine (Italia), del cruce sauvignon blanc $\times 20 / 3$, y registrada en 2015 en el Registro Nazionale delle Varietà di Vite [16]. La segunda variedad PIWI fue souvignier gris (C.v. FR 392-83), se obtuvo por el Staatliches Weinbauinstitut Freiburg (Alemania) en 1983, por cruce de cabernet sauvignon (sauvignon blanc $\times$ cabernet franc) con bronner (merzling $\times$ Gm 6494 (zarya severa $\times$ st. laurent)). Registrada en la German Federal Office of Varietal Registration (Bundessortenamt) en 2008 [17] y en Francia en el "Catalogue des variétés de vigne" [18]. La tercera variedad PIWI, muscaris (C.v. FR 493-87) se obtuvo en 1987 también por el Staatliches Weinbauinstitut Freiburg del cruce de solaris $\times$ muscat, y no se conocen datos de registro [19]. Las plantas del estudio están certificadas por el vivero Vivai Cooperativi Rauscedo (Rauscedo, Italia) en el caso de sauvignon kretos, y por el vivero Pepinières Viticoles Mercier (Vix, Francia) para las otras dos variedades. Se han empleado 224 cepas de variedades PIWI: 69 de sauvignon kretos, 76 de souvignier gris y 79 de muscaris, todas ellas plantadas durante el mes de abril de 2015 en forma de planta injerto, sobre patrón SO4. Para propósitos de comparación, se ha utilizado como variedad testigo y tratada (183 plantas) sauvignon blanc (clon 376), injertado en $\mathrm{SO} 4$.

\subsection{Diseño experimental y técnicas culturales}

Las variedades a estudiar se distribuyeron en dos parcelas contiguas, con filas de plantas testigo ubicadas a ambos lados de las resistentes y seguidamente las filas de sauvignon blanc tratadas. Las cepas están plantadas en doble fila de unos $40 \mathrm{~m}$, con marco de plantación de 2,8 $\times$ 0,9 m (densidad de plantación de 3968 plantas/ha), utilizando un sistema de formación con estructura de soporte y vegetación libre dirigida a mano (sistema Cortina), con una altura de formación de 1,4 m.

Las plantas fueron sometidas a unas condiciones exigentes respecto a rendimiento, forzando algunos aspectos de manejo (riego, hierba, canopy) para evaluar realmente la resistencia de los cultivares experimentales. 


\section{CONGRESO IBÉRICO DE AGROINGENIERÍA \\ X CONGRESSO IBÉRICO DE AGROENGENHARIA \\ 3 - 6 septiembre 2019, Huesca - España}

El riego se realizó con mangueras de goteo integrado cada $75 \mathrm{~cm}$ y con un caudal de $4 \mathrm{~L} / \mathrm{h}$. Las mangueras están elevadas $50 \mathrm{~cm}$ sobre el suelo.

Todas las plantas (tanto de las variedades resistentes como testigo y tratadas) tuvieron que ser tratadas frente a la erinosis en la campaña 2016, por estar muy afectadas (al ser plantas jóvenes el efecto del ataque es mayor). Por lo demás, en las PIWI y en las filas testigo no se han aplicado tratamientos para plagas o enfermedades. Respecto a los fungicidas aplicados en las líneas tratadas, para mildiu se ha tratado con un fungicida preventivo (hidróxido cúprico) y uno sistémico (metalaxil + mancozeb); y para oídio con miclobutanil como fungicida preventivo, y con varios fungicidas sistémicos (bupirimato, tebuconazol y proquinazid).

\subsection{Recogida de datos}

El seguimiento climático se realizó mediante placas "Moisture meter datalogger" de Hobby Boards [20], con sensores de humedad en hoja y temperatura, situadas en la parcela de estudio, complementando las medidas con datos climáticos de la Confederación Hidrográfica del Ebro (CHE) y del Sistema de Información Agroclimática para el Regadío (SIAR).

Se realizó un seguimiento semanal en campo de todas las variedades desde marzo hasta septiembre para las tres campañas. Se han monitorizado los estados fenológicos conforme a las siguientes escalas: Código Eichhorn-Lorenz [21], Estados fenológicos de la vid [22] y la Codificación $\mathrm{BBCH}$ de los estadios fenológicos de desarrollo de la vid [23]. Se ha realizado también un seguimiento de los principales caracteres ampelográficos tomando 10 elementos de referencia y utilizando para ello la lista de descriptores de la Organización Internacional de la Viña y el Vino (OIV) para variedades de vid y especies Vitis [24]. Los descriptores utilizados han sido para hoja joven, hoja adulta, flor, racimo, baya, producción y enfermedad. La caracterización ampelográfica para la variedad sauvignon blanc se realizó sobre las líneas testigo.

Para la monitorización de enfermedades, cada semana se observó una muestra de 55 órganos elegidos al azar en cada bloque, y en su momento, 25 racimos. Para la evaluación de los parámetros relativos a la presencia y ataque de dichas enfermedades, se ha seguido la propuesta marcada por la Organización Europea y Mediterránea de Protección Vegetal (EPPO) en 2009. En ésta se establece una escala en base al porcentaje de área del órgano afectada por la enfermedad [25]. Para calcular el grado de ataque se ha utilizado la fórmula de Townsend-Heuberger, y para la evaluación de la eficacia del tratamiento (relación entre el grado de ataque en las cepas testigo frente a las tratadas), la fórmula de Abbott [26].

La vendimia se realizó de forma manual. En la campaña 2017 se realizó en una única fecha para las tres variedades (22 de agosto), mientras que en la campaña 2018 se repartió en dos fechas: 22 de agosto para muscaris y 3 de septiembre para sauvignon kretos y souvignier gris.

Para la evaluación del equilibrio de la planta entre producción y desarrollo se ha empleado el índice de Ravaz [27], en base a datos de peso de la cosecha y de peso de la madera de poda.

\subsection{Vinificación}

Se procesaron $200 \mathrm{~kg}$ de uva por variedad. Se realizó un prensado sin despalillar, con adición de enzima pectolítica (Pectazina, $3 \mathrm{~g} / 100 \mathrm{~kg}$ ) y metabisulfito potásico (30 ppm de $\mathrm{SO}_{2}$ ), con un posterior desfangado de $24 \mathrm{~h}$ en frío. Tras el trasiego, se realizó el aporte de levaduras (Saccharomyces bayanus a $20 \mathrm{~g} / \mathrm{hL}$ ), y se procedió al seguimiento y control de la fermentación alcohólica, terminando esta al cabo de 13 días (muscaris), 20 días (sauvignon kretos) y 16 días (souvignier gris). Tras un trasiego final y sulfitado (metabisulfito, $4,5 \mathrm{~g} / \mathrm{hL}$ ), se procedió al análisis de los vinos terminados. 
X CONGRESO IBÉRICO DE AGROINGENIERÍA

X CONGRESSO IBÉRICO DE AGROENGENHARIA

3- 6 septiembre 2019, Huesca - España

\section{Resultados y discusión}

\subsection{Datos climáticos}

Las temperaturas y precipitaciones medias mensuales para el periodo de estudio (marzoseptiembre) en las tres campañas se recogen en la Tabla 1. Las campañas 2016 y 2017 correspondieron a años secos (243,6 y 294,3 mm, respectivamente), si bien el 2016 presentó una distribución de lluvias más irregular; la campaña 2018 tuvo precipitaciones por encima de la media (499 mm).

Tabla 1. Arriba: temperaturas medias mensuales $\left({ }^{\circ} \mathrm{C}\right)$; abajo: precipitaciones medias mensuales $(\mathrm{mm})$ para las tres campañas.

\begin{tabular}{cccccccc}
\hline Campaña & Marzo & Abril & Mayo & Junio & Julio & Agosto & Septiembre \\
\hline 2016 & 9,3 & 12,5 & 16,1 & 21,6 & 24,9 & 24,0 & 21,4 \\
2017 & 11,8 & 13,5 & 18,2 & 23,7 & 24,8 & 24,3 & 18,7 \\
2018 & 8,9 & 13,4 & 17,1 & 22,0 & 25,6 & 25,2 & 22,2 \\
\hline \hline Campaña & Marzo & Abril & Mayo & Junio & Julio & Agosto & Septiembre \\
\hline 2016 & 69,2 & 93,0 & 33,0 & 13,6 & 11,8 & 1,0 & 22,0 \\
2017 & 109,4 & 34,0 & 45,4 & 47,8 & 23,3 & 7,0 & 27,4 \\
2018 & 60,7 & 117,0 & 142,6 & 28,4 & 13,0 & 101,9 & 35,4 \\
\hline
\end{tabular}

\subsection{Fenología y ampelografía}

Los resultados obtenidos en campo se ajustaron a lo establecido en las fichas varietales para la mayor parte de descriptores en las tres campañas. Las pequeñas diferencias detectadas, sobre todo en la campaña 2016, deben ser atribuidas a expresión de juvenilidad. En general, se observa que estas variedades tienen un comportamiento similar a las cultivadas en la D.O. Somontano y no presentaron problemas de adaptación.

\subsection{Monitorización de enfermedades}

\subsubsection{Oídio}

En la campaña 2016, y en concreto el 5 de julio, se observaron ataques muy leves en hoja en las variedades testigo y tratadas (en las resistentes no se produjo ataque). El ataque en racimo apareció la semana del 19 de julio (Figura 1a) y resultó significativo en las variedades testigo, en las que se alcanzó aproximadamente un $20 \%$ de ataque (en las variedades resistentes no se apreció racimo alguno afectado).

En la campaña 2017 no hubo ataque de oídio en las PIWI, pero sí en las variedades testigo y tratadas, tanto en hoja (Figura 1b) como en racimo (Figura 1c).

En la campaña 2018 no se detectó ataque en hojas de testigos, tratadas o PIWI, pero sí en racimos (Figura 1d). En sauvignon kretos se registró un bajo grado de ataque (un total de 13 anotaciones, todas con intensidad de ataque $<25 \%$ ) del 26 de julio al 16 de agosto; en souvignier gris el grado de ataque fue aún menor ( 6 anotaciones, todas con intensidad de ataque $<25 \%$ ) del 3 al 16 de agosto; y en muscaris fue más alto, con 20 anotaciones con intensidad de ataque $<25 \%$ (del 3 al 16 de agosto) y sólo una en el rango de intensidad de ataque del 25-50\% en la última medida (16 de agosto). Comparativamente, los grados de ataque en plantas testigo y tratadas fueron notablemente más altos. 

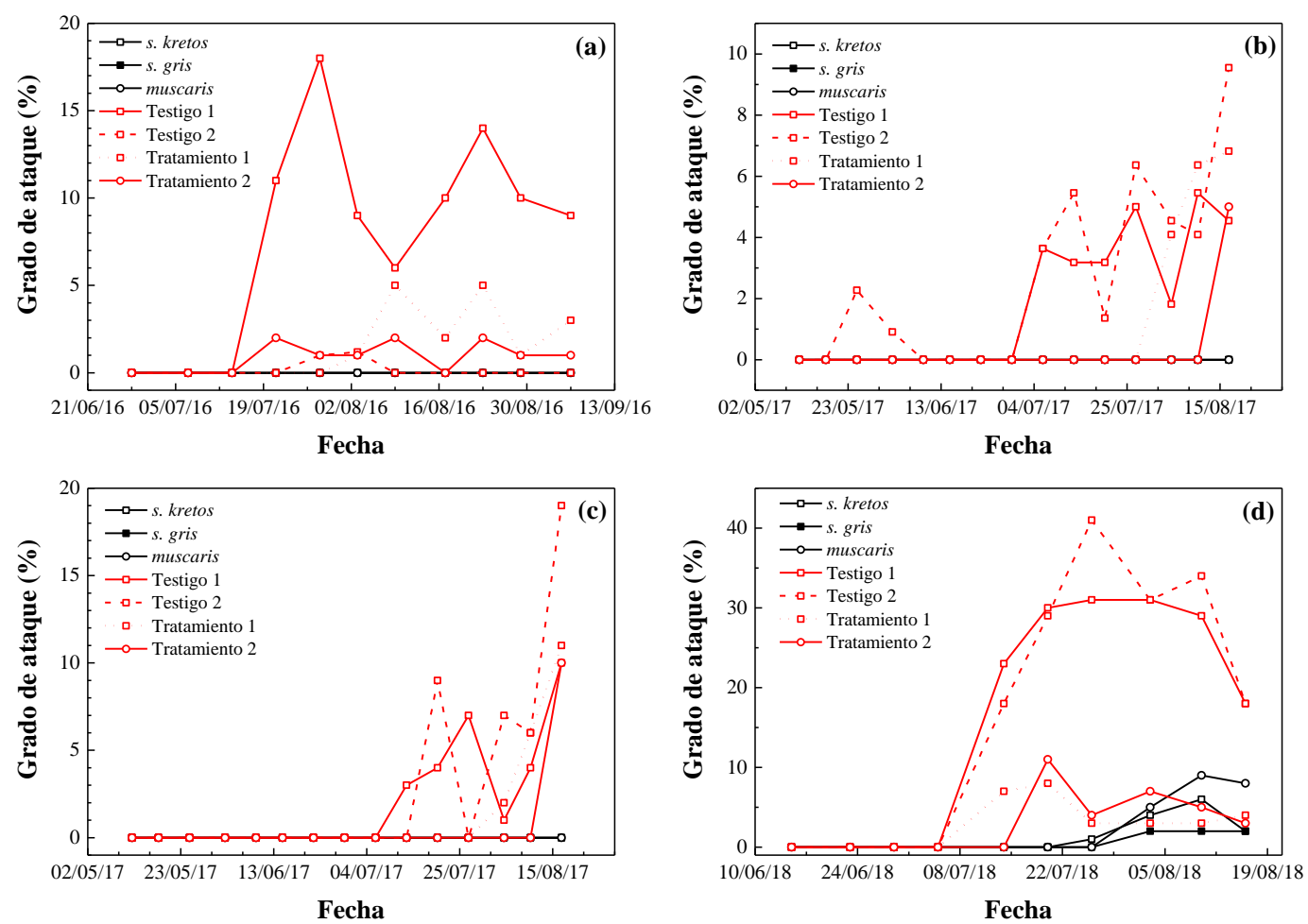

Figura 1. Evolución temporal del grado de ataque de oídio en: (a) campaña 2016 en racimo; (b) campaña 2017 en hoja; (c) campaña 2017 en racimo; y (d) campaña 2018 en racimo.

\subsubsection{Mildiu}

En la campaña 2016 se detectaron daños en hoja en todas las cepas. Las variedades resistentes presentaron un bajo grado de ataque, siendo las cepas testigo y tratadas las más afectadas. En cuanto a los daños en racimo, sólo se detectaron para las cepas testigo y tratadas: del 28 de junio al 12 de julio se observaron racimos totalmente destruidos antes de alcanzar el tamaño de baya guisante, seguido de otro ataque que afectó a racimos de mayor tamaño, en los cuales, con el paso de las semanas, se observó el desecamiento de los granos.

En la campaña 2017 no se detectaron daños en ninguna de las variedades PIWI, ni en las viñas tratadas ni en la variedad testigo 2 . Anecdóticamente, se detectaron intensidades de ataque leves $(<25 \%)$ en hoja para la variedad testigo 1 en la recogida de datos del 13 de julio (3 de 55 muestras, 5.77\%). En racimo no se detectaron daños.

En el año 2018 sí se detectó ataque, tanto en hoja como en racimo. En cuanto al ataque en hoja, en sauvignon kretos se detectaron 22 casos, con intensidad de ataque $<25 \%$, entre el 15 de junio y el 5 de julio; en souvignier gris se detectaron un total de 17 casos, todos con intensidad de ataque $<25 \%$, entre el mismo periodo; y en muscaris se detectaron un total de 15 casos, con intensidad de ataque $<25 \%$, también entre el 15 de junio y el 5 de julio. En cuanto a los daños en racimo, en sauvignon kretos se detectó un único caso, con intensidad de ataque $<25 \%$, el 5 de julio; en souvignier gris se detectaron un total de 7 casos, todos con intensidad de ataque $<25 \%$, entre el 15 de junio y el 5 de julio; y en muscaris se detectaron dos casos, con intensidad de ataque $<25 \%$, entre el 29 de junio y el 5 de julio. Los daños en plantas testigo y tratadas fueron más altos, llegando a un $60 \%$ de grado de ataque. 

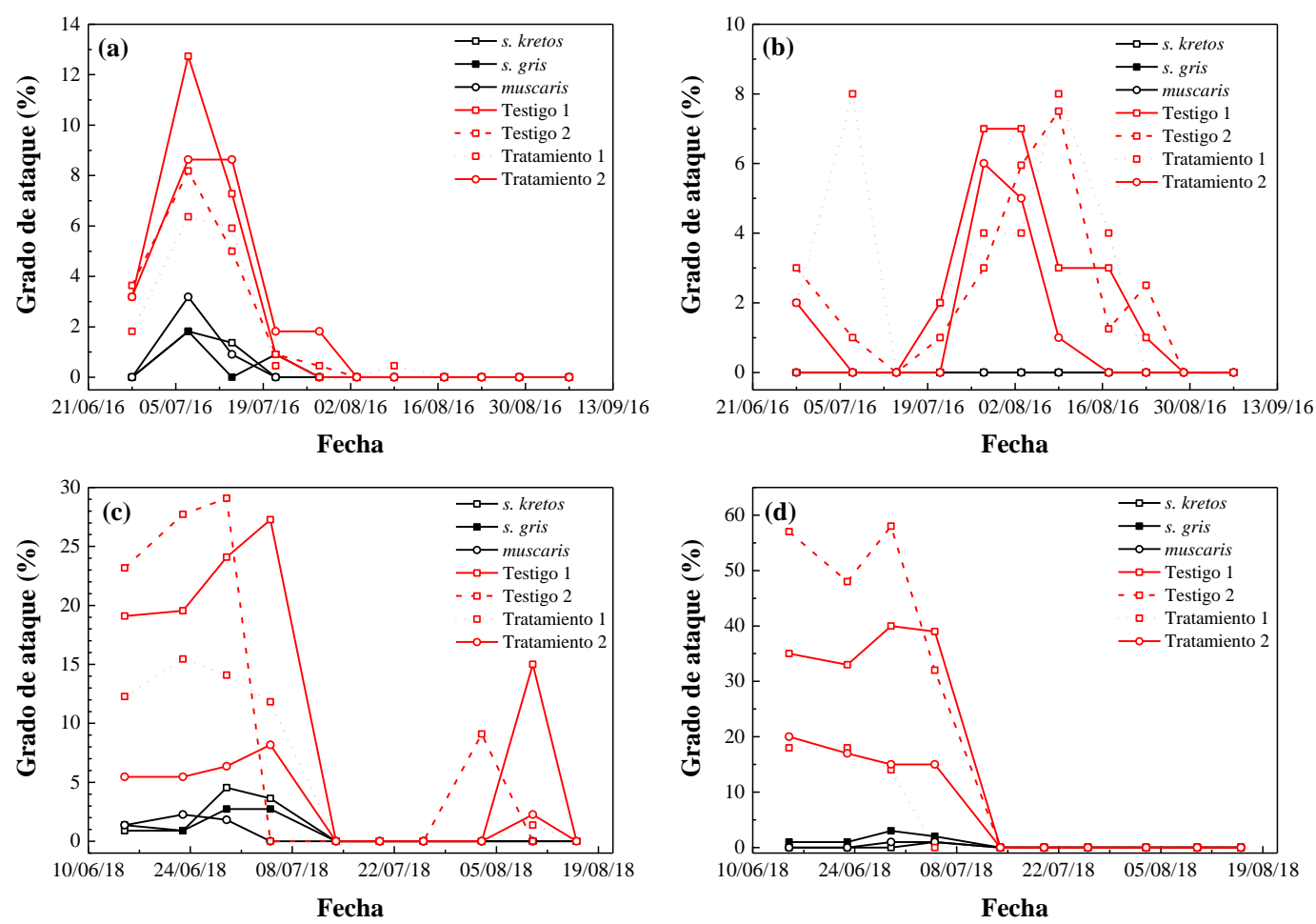

Figura 2. Evolución temporal del grado de ataque de mildiu en las campañas 2016 (arriba) y 2018 (abajo), en hoja (izquierda) y en racimo (derecha).

\subsubsection{Podredumbre gris}

En la campaña 2016 (Figura 3a) se detectaron ataques a finales del mes de agosto en las cepas testigo y tratadas, sin detectarse ningún síntoma en las PIWI. Los racimos que presentaron daño fue en su mayoría con un 25-50\% de intensidad de ataque. De forma similar, en la campaña 2017 (Figura $3 b$ ) no hubo ataque en las variedades PIWI. En las cepas testigo y tratadas se detectaron daños puntuales en racimo el 10 y el 17 de agosto (con intensidad de ataque de hasta el 50\%), que afectaron a un porcentaje inferior al 4\% de los racimos muestreados. En la campaña 2018 (no mostrada) no se detectaron daños por podredumbre gris en ningún caso (ni en plantas testigo, ni en tratadas, ni en las PIWI).
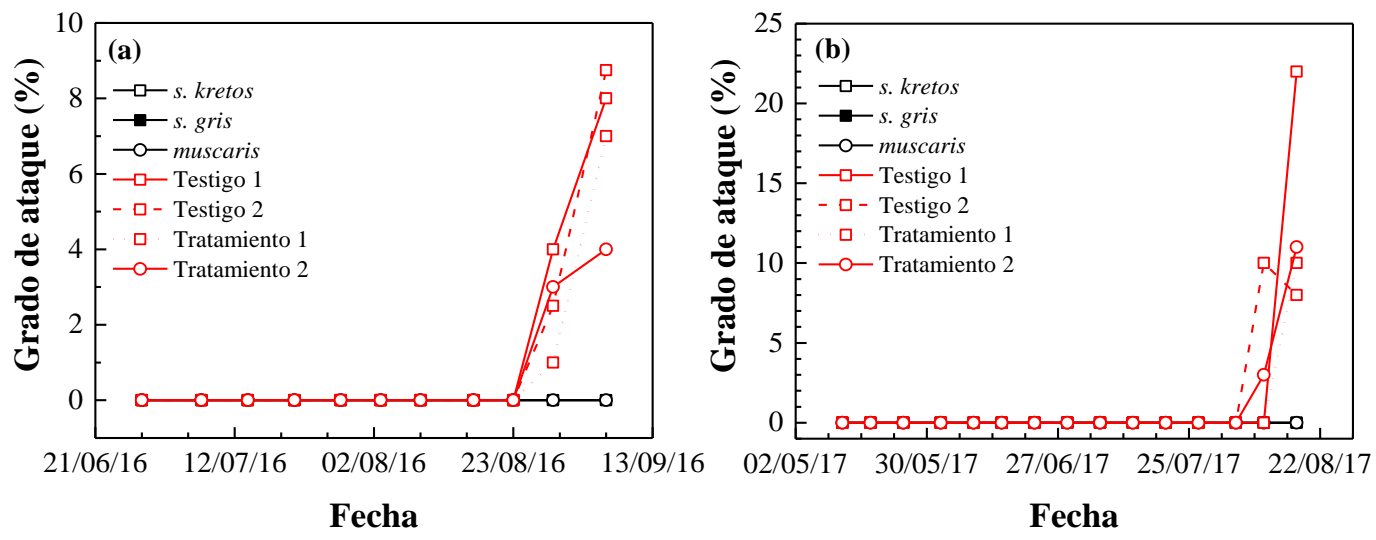

Figura 3. Evolución temporal del grado de ataque de podredumbre gris en las campañas 2016 (a) y 2017 (b). 


\section{CONGRESO IBÉRICO DE AGROINGENIERÍA \\ X CONGRESSO IBÉRICO DE AGROENGENHARIA \\ 3 - 6 septiembre 2019, Huesca - España}

\subsection{Eficacias de tratamiento}

En la campaña 2016, las eficacias de tratamiento frente a mildiu fueron muy bajas (18\%) en hoja y algo superiores en racimo (47\%); en el caso del oídio, se observaron eficacias medias de tratamiento superiores al 50\%, tanto en hoja como en racimo. En la campaña 2017, las eficacias de tratamiento contra oídio fueron superiores al $60 \%$ en hoja (alcanzándose un $93 \%$ en la parcela 2). En la campaña 2018, la eficacia media de tratamiento frente a mildiu fue próxima a un $65 \%$, tanto en hoja como en racimo; y la eficacia media contra oídio en racimo fue de hasta un $82 \%$.

\subsection{Producción e indices de Ravaz}

En base a los datos de producción y de poda, en la Tabla 2 se recogen los índices de Ravaz calculados. Los valores recomendados son 5-7 si se quiere evitar desequilibrios en el viñedo, si bien otros autores plantean un rango más amplio (5-12), en función del cultivar, carga, dosis de riego y sistemas de conducción [28,29]. Valores muy altos, por encima de 10, nos indican que la viña ha producido mucha uva para su vigor, es decir, que presenta un desajunte por exceso de producción o defecto de vigor. Por el contrario, valores por debajo de 3 nos indicarían un vigor excesivo del viñedo, es decir, una baja productividad para esas condiciones de fertilidad. Por tanto, se deduce que para las dos campañas con datos de producción (2017 y 2018), se produjeron desequilibrios importantes para souvignier gris en 2017 y muscaris en 2018, si bien pueden justificarse por la juventud de la plantación.

Tabla 2. Datos de producción y poda, e índices de Ravaz calculados.

\begin{tabular}{ccccccc}
\hline \multirow{2}{*}{ Variedad } & \multicolumn{3}{c}{ Campaña 2017 } & \multicolumn{3}{c}{ Campaña 2018 } \\
\cline { 2 - 7 } & $\begin{array}{c}\text { Producción } \\
\text { (kg/cepa) }\end{array}$ & $\begin{array}{c}\text { Peso poda } \\
\text { (kg/cepa) }\end{array}$ & $\begin{array}{c}\text { Índice } \\
\text { de Ravaz }\end{array}$ & $\begin{array}{c}\text { Producción } \\
\text { (kg/cepa) }\end{array}$ & $\begin{array}{c}\text { Peso poda } \\
\text { (kg/cepa) }\end{array}$ & $\begin{array}{c}\text { Índice } \\
\text { de Ravaz }\end{array}$ \\
\hline sauvignon kretos & 3,50 & 0,86 & 4,05 & 3,27 & 0,80 & 4,10 \\
souvignier gris & 2,03 & 0,15 & 13,67 & 2,27 & 0,49 & 4,62 \\
muscaris & 1,48 & 0,17 & 8,62 & 2,01 & 0,12 & 16,41 \\
\hline
\end{tabular}

\subsection{Valores iniciales de los mostos}

Los resultados de las analíticas realizadas a los mostos a la entrada a la bodega (22/08/2017 para las tres PIWI; 22/08/2018 para muscaris, 03/09/2018 para sauvignon kretos y souvignier gris) se recogen en la Tabla 3. El pH y grado alcohólico probable se encuentra en los datos medios de otras variedades cultivadas en el territorio, aunque la variedad muscaris alcanza un grado alcohólico muy alto y una acidez total demasiado baja. La variedad souvignier gris es la única que manifiesta deficiencias en el nitrógeno fácilmente asimilable, lo que plantearía problemas en la fermentación, si no se corrige.

Tabla 3. Valores iniciales de los mostos en las campañas 2017 y 2018 para las tres variedades PIWI.

\begin{tabular}{ccccccc}
\hline \multirow{2}{*}{ Variable } & \multicolumn{2}{c}{ sauvignon kretos } & \multicolumn{2}{c}{ souvignier gris } & \multicolumn{2}{c}{ muscaris } \\
\cline { 2 - 7 } & $\mathbf{2 0 1 7}$ & $\mathbf{2 0 1 8}$ & $\mathbf{2 0 1 7}$ & $\mathbf{2 0 1 8}$ & $\mathbf{2 0 1 7}$ & $\mathbf{2 0 1 8}$ \\
\hline Acidez total (g/L) & 6,61 & 6,1 & 6,08 & 5,5 & 3,79 & 3,9 \\
$\mathrm{pH}$ & 3,14 & 3,16 & 3,35 & 3,38 & 3,15 & 3,71 \\
Grado alcohólico probable & 12,9 & 13,66 & 14,3 & 14,96 & 16,2 & 15,60 \\
NFA & 223 & 100 & 190 & 84 & 165 & 224 \\
\hline
\end{tabular}

NFA: nitrógeno fácilmente asimilable 


\section{CONGRESO IBÉRICO DE AGROINGENIERÍA \\ X CONGRESSO IBÉRICO DE AGROENGENHARIA \\ 3 - 6 septiembre 2019, Huesca - España}

\subsection{Resultados de la vinificación}

Los vinos obtenidos se sometieron a un análisis sensorial en el Laboratorio de análisis del aroma y enología (LAAE) de Zaragoza. El vino resultante de la variedad muscaris -con descriptores de almendra amarga, fruto seco, terpénico en alta intensidad- fue el que recibió una mejor acogida en ambos años. El de sauvignon kretos puede llegar a tener una aceptación similar al de la variedad estándar (sauvingnon blanc) con solo modular la acidez y algunas notas desagradables, objetivo alcanzable tras realizar pruebas de vinificación con distintos estados de maduración. En cuanto al vino de la variedad souvignier gris, los resultados no fueron concluyentes: si bien el vino de la campaña 2017 tuvo una aceptación muy baja, su aceptación en la cata realizada para la microvinificación de la campaña 2018 fue buena. En los tres casos se destacó una gran intensidad aromática de los vinos, con aromas poco relacionados con alimentos.

\section{Conclusiones}

De la comparación de los resultados obtenidos para cada una de las enfermedades y variedades con las fichas varietales de éstas, se ha podido concluir de forma general que las tres variedades PIWI ensayadas muestran resistencia alta para mildiu, oídio e incluso para podredumbre gris (para la que se establecía un nivel medio de resistencia en las fichas varietales). La variedad sauvignon kretos ha exhibido un rendimiento de cepa superior al control, mientras que souvignier gris y muscaris presentaron rendimientos menores. No obstante, la variedad muscaris puede ser la más interesante cara a producción a escala piloto, por su buen perfil organoléptico. Estas variedades plantean una alternativa de producción en entornos sometidos a presión fúngica y pueden ocupar el nicho de mercado de consumidores que valoran la reducción de fitosanitarios en la viticultura.

\section{Referencias}

1. Galet, P. Précis De Pathologie Viticole. 3 ed.; Imprimerie JF Impression: Montpellier, France, 1999; p 296.

2. Piwi-International. 2016, 2616.

3. Rousseau, J.; Chanfreau, S.; Bontemps, É. Les Cépages Résistants and Maladies Cryptogamiques. Groupe ICV: Bordeaux, France, 2013; p 228.

4. Sivcev, B.; Sivcev, I.; Rankovic-Vasic, Z. Natural process and use of natural matters in organic viticulture. Journal of Agricultural Sciences, Belgrade 2010, 55, 195-215.

5. Pavloušek, P.; Kumšta, M.; Mateiciucová, P. Adaptation of New Resistant Grapevine Varieties to the Terroir in the Czech Republic. In X Congrès Internationaux des Terroirs Vitivinicoles, Society of International Terroir Experts in vitiviniculture: Tokaj-Eger, Hungary, 2014; pp 1-5.

6. Siegfried, W.; Temperli, T. Piwi-Reben im vergleich-ein zwischenbericht. Schweiz. Z. Obst Weinbau 2008, 17, 6-9.

7. Van der Meer, M.; Weibel, F.; Levite, D.; Häseli, A.; Vombach, D. Acceptation des vins de cépages résistants par les consommateurs. Revue suisse de viticulture, arboriculture et horticulture 2010, 42, 147-150.

8. Becker, A. Piwis in der Praxis. Schweiz. Z. Obst Weinbau 2013, 3, 4-7.

9. Galbrun, C. Etude Inra - Comment réduire ses coûts de production de 50\%. https://www.reussir.fr/vigne/comment-reduire-ses-couts-de-production-de-50

10. Fuller, K.B.; Alston, J.M.; Sambucci, O.S. The value of powdery mildew resistance in grapes: Evidence from California. Wine Economics and Policy 2014, 3, 90-107.

11. Reynolds, A.G.; Vanden Heuvel, J.E. Influence of Grapevine Training Systems on Vine Growth and Fruit Composition: A Review. American Journal of Enology and Viticulture 2009, 60, 251-268.

12. Barthe, $\mathrm{C}$. Impact de la charge fruitière sur la maturité et la qualité du raisin chez le Seyval blanc et le Vandal-Cliche, deux cépages hybrides cultivés au Québec. Université Laval, Québec, Canada, 2015.

13. Sun, Q.; Sacks, G.; Lerch, S.; Vanden Heuvel, J.E. Impact of Shoot Thinning and Harvest Date on Yield Components, Fruit Composition, and Wine Quality of Marechal Foch. American Journal of Enology and Viticulture 2011, 62, 32-41. 


\section{CONGRESO IBÉRICO DE AGROINGENIERÍA \\ $X$ CONGRESSO IBÉRICO DE AGROENGENHARIA \\ 3 - 6 septiembre 2019, Huesca - España}

14. Lissarrague, J.R.; Baeza, P.; Peiro, E.; Ayuso, J.M.; Cibriain, F.; Blanco, J.A.; Villalba, P. Híbridos resistentes a mildiu-oidio: la apuesta por la sostenibilidad y el respeto medioambiental de VCR. Olint 2016, pp 6-14.

15. Badía, D.; Cuchí, J.; Martí, C.; Casanova, J. Los suelos de los viñedos en la D.O. Somontano. Prensas Universitarias de Zaragoza: Zaragoza, Spain, 2006; Vol. 8, p 199.

16. Morgante, M.; Testolin, R. Nuovi vitigni resistenti alle malattie. In Quaderni Tecnici VCR, Rauscedo, V.C., Ed. Italia, 2016; Vol. 15, p 40.

17. Pritchard, D. Souvignier gris (originally known as Fr. 392-83). http://www.winegrowers.info/varieties/Vine_varieties/Souvignier\%20gris.htm

18. Bonnet, P.; Lacombe, T. Le catalogue des vignes cultivées en France. http://plantgrape.plantnetproject.org/es/nouvelles

19. Pritchard, D. Muscaris (originally known as Fr. 493-87). http://www.winegrowers.info/varieties/Vine_varieties/Muscaris.htm

20. Vickery, E. Hobby boards moisture meter datalogger. Instruction manual and technical specifications. Hobbyboards, Ed. EEUU, 2012; p 14.

21. Eichhorn, K.W.; Lorenz, D. Phaenologische Entwicklungsstadien der Rebe: Anwendungstermine $d$. Pflanzenschutzmittel; Sonderdr. aus "Der deutsche Weinbau". BASF: 1978.

22. Baillod, M.; Baggiolini, M. Les stades repères de la vignee. Revue suisse de Viticulture, Arboriculture, Horticulture 1993, 25, 7-9.

23. Lorenz, D.; Eichhorn, K.; Bleiholder, H.; Klose, R.; Meier, U.; Weber, E. Phenological growth stages of the grapevine (Vitis vinifera L. ssp. vinifera)-Codes and descriptions according to the extended $\mathrm{BBCH}$ scale. Australian Journal of Grape and Wine Research 1995, 1, 100-103.

24. OIV. Lista de descriptores OIV para variedades de vid y especies vitis. $2^{\circ}$ ed.; vin, O.I.d.l.v.e.d., Ed. Paris, 2001; p 179.

25. Townsend, G.R.; Heuberger, J.W. Methods for estimating losses caused by diseases in fungicide experiments. The Plant Disease Reporter 1943, 24, 340-343.

26. Abbott, W.S. Abbott's formula. Journal of Econonomic Entomology 1925, 18, 267-268.

27. Vasconcelos, M.C.; Castagnoli, S. Leaf Canopy Structure and Vine Performance. American Journal of Enology and Viticulture 2000, 51, 390-396.

28. Smart, R.; Robinson, M. Sunlight into wine: a handbook for winegrape canopy management. Winetitles: Adelaide, Australia, 1991; p 88.

29. Matthews, M.A. Terroir and other myths of winegrowing. University of California Press: Oakland, California, 2015; p xii, 308 pages. 